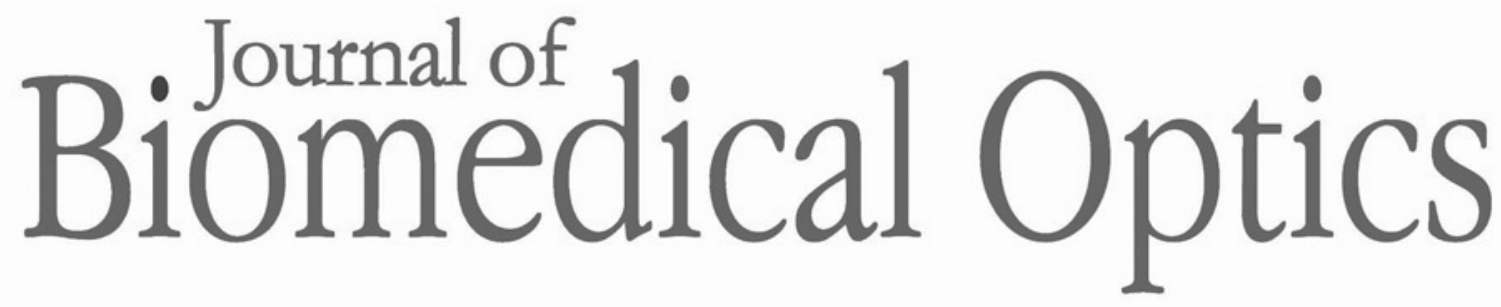

\title{
Applicability of quantitative optical imaging techniques for intraoperative perfusion diagnostics: a comparison of laser speckle contrast imaging, sidestream dark-field microscopy, and optical coherence tomography
}

Sanne M. Jansen

Daniel M. de Bruin

Dirk J. Faber

Iwan J. G. G. Dobbe

Erik Heeg

Dan M. J. Milstein

Simon D. Strackee

Ton G. van Leeuwen 


\title{
Applicability of quantitative optical imaging techniques for intraoperative perfusion diagnostics: a comparison of laser speckle contrast imaging, sidestream dark-field microscopy, and optical coherence tomography
}

\author{
Sanne M. Jansen, ${ }^{a, b, *}$ Daniel M. de Bruin, ${ }^{a, c}$ Dirk J. Faber, ${ }^{a}$ Iwan J. G. G. Dobbe, ${ }^{a}$ Erik Heeg, ${ }^{b}$ \\ Dan M. J. Milstein, ${ }^{d}$ Simon D. Strackee, ${ }^{b}$ and Ton G. van Leeuwen ${ }^{a}$ \\ anniversity of Amsterdam, Academic Medical Center, Department of Biomedical Engineering and Physics, Amsterdam, The Netherlands \\ bUniversity of Amsterdam, Academic Medical Center, Department of Plastic, Reconstructive, and Hand Surgery, Amsterdam, The Netherlands \\ 'University of Amsterdam, Academic Medical Center, Department of Urology, Amsterdam, The Netherlands \\ dUniversity of Amsterdam, Academic Medical Center, Department of Oral and Maxillofacial Surgery, Amsterdam, The Netherlands
}

\begin{abstract}
Patient morbidity and mortality due to hemodynamic complications are a major problem in surgery. Optical techniques can image blood flow in real-time and high-resolution, thereby enabling perfusion monitoring intraoperatively. We tested the feasibility and validity of laser speckle contrast imaging (LSCl), optical coherence tomography (OCT), and sidestream dark-field microscopy (SDF) for perfusion diagnostics in a phantom model using whole blood. Microvessels with diameters of 50,100 , and $400 \mu \mathrm{m}$ were constructed in a scattering phantom. Perfusion was simulated by pumping heparinized human whole blood at five velocities (0 to $20 \mathrm{~mm} / \mathrm{s}$ ). Vessel diameter and blood flow velocity were assessed with LSCI, OCT, and SDF. Quantification of vessel diameter was feasible with OCT and SDF. LSCl could only visualize the $400-\mu \mathrm{m}$ vessel, perfusion units scaled nonlinearly with blood velocity. OCT could assess blood flow velocity in terms of inverse OCT speckle decorrelation time. SDF was not feasible to measure blood flow; however, for diluted blood the measurements were linear with the input velocity up to $1 \mathrm{~mm} / \mathrm{s}$. LSCI, OCT, and SDF were feasible to visualize blood flow. Validated blood flow velocity measurements intraoperatively in the desired parameter $\left(\mathrm{mL} \cdot \mathrm{min}^{-1} \cdot \mathrm{g}^{-1}\right)$ remain challenging. ๑ 2017 Society of Photo-Optical Instrumentation Engineers (SPIE) [DOI: 10.1117/1.JBO.22.8.086004]
\end{abstract}

Keywords: biomedical optical imaging; medical diagnostics imaging; perfusion; phantom; laser speckle contrast imaging; optical coherence tomography; sidestream dark-field microscopy.

Paper 160688R received Nov. 15, 2016; accepted for publication Jul. 13, 2017; published online Aug. 19, 2017.

\section{Introduction}

Cell function and viability is highly dependent on the effective diffusion and exchange of oxygen, nutrients, and metabolic waste between circulating blood and tissue. Restriction of blood supply by hemodynamic change results in a lack of oxygen and nutrients that often culminates in tissue necrosis. ${ }^{1}$ Accordingly, compromised perfusion during surgery is thought to be associated with major complications. ${ }^{2}$ In free-flap surgery, tissue is transferred from the donor site to the recipient site in case of tissue loss due to trauma or malignant disease. After transplantation, blood vessels are reconnected to sustain blood supply in the grafted tissue. Due to vascular compromise, these flaps may become necrotic and, in $\sim 4 \%$ of patients, total flap loss occurs resulting in high morbidity and reoperation rates. ${ }^{3}$ In gastrointestinal surgery, for example, esophageal or bowel tissue is reconnected after the excision of malignant tissue yielding leakage with high rates of morbidity (10\% to $30 \%$ ) and mortality (4\%). ${ }^{4}$ Although monitoring tissue perfusion is reasonable in view of procedural vascular compromise, this is not current practice. Monitoring tissue perfusion provides the

*Address all correspondence to: Sanne M. Jansen, E-mail: s.m.jansen@amc. uva.nl opportunity to change or modify surgical treatment and to guide anesthesiologists in optimizing physiological stability by customizing fluid administration or medication. Therefore, the utilization of validated perfusion imaging techniques during surgery may decrease morbidity and mortality associated with poor tissue hemodynamics. Quantifying perfusion in terms of flow per unit of tissue mass $\left(\mathrm{mL} \cdot \mathrm{min}^{-1} \cdot \mathrm{g}^{-1}\right)$ is possible with positron emission tomography (PET) ${ }^{5}$ computed tomography (CT), ${ }^{6}$ and magnetic resonance imaging (MRI). ${ }^{7}$ However, for most hospitals, PET, CT, and MRI are not feasible or financially viable for measuring perfusion intraoperatively. For clinical implementation, readily and commercially available low cost, noninvasive alternatives are desired.

Optical monitoring and imaging techniques have emerged as powerful diagnostic tools in different fields of medicine, such as ophthalmology for visualizing retinal vasculature, ${ }^{8}$ anesthesiology for diagnosing shock in critically ill patients, ${ }^{9}$ and neurology for cerebral perfusion imaging. ${ }^{10}$ Due to their minimally invasive character and real-time visualization with high-contrast, these techniques could be of great advantage in intraoperative settings. Laser speckle contrast imaging (LSCI), ${ }^{11}$ sidestream 
dark-field microscopy (SDF), ${ }^{12}$ and optical coherence tomography $(\mathrm{OCT})^{13}$ are readily and commercially available noninvasive techniques. LSCI quantifies laser speckle fluctuations that vary with particle motion. OCT quantifies localized speckle fluctuations, related to blood flow velocity. SDF illuminates and records red blood cells in flow and quantifies motion by tracking RBC position through subsequent images. However, neither of these techniques is presently capable of quantifying perfusion in terms of flow per unit of tissue mass standard units $\left(\mathrm{mL} \cdot \min ^{-1} \cdot \mathrm{g}^{-1}\right)$ and vary with their imaging resolution and field of view (FOV).

Following the idea-development-exploration-assessmentlong-term study recommendations for evaluation of new surgical technologies, ${ }^{14}$ a translational phantom-to-patient study is needed in which these techniques can be validated in a controlled in vitro setting. For the clinician, it is important to know the accuracy of measurements and to be familiar with possible sources of error. Therefore, the objective of this study was to investigate the feasibility and validity of LSCI, OCT, and SDF to visualize and quantify perfusion-related parameters, such as blood flow $(\mathrm{mL} / \mathrm{min})$, velocity $(\mathrm{m} / \mathrm{s})$, and vessel diameter $(\mu \mathrm{m})$ in a phantom model. In this study, whole blood was used instead of intralipid, in contrast to most studies, to ensure realistic optical properties of blood vessels in a tissue-like phantom with the optical properties of human soft tissue. We investigated the feasibility and validity of assessing vessel diameter and blood flow velocity and discussed weaknesses and strengths of using these imaging modalities in an intraoperative setting.

\section{Materials and Methods}

\subsection{Setup}

Human perfusion was simulated by pumping heparinized human whole blood obtained from a healthy volunteer through a flow phantom containing microvessels with lumen diameters equal to 50, 100, and $400 \mu \mathrm{m}$ (Fig. 1).

\subsubsection{Phantom}

Using Solid Works (Dassault Systèmes SOLIDWORKS Corp., Waltham, Massachusetts), we designed two silicone phantoms with embedded vessels. The first phantom (phantom A) contained a 50- and 100- $\mu \mathrm{m}$-diameter vessel, positioned under an

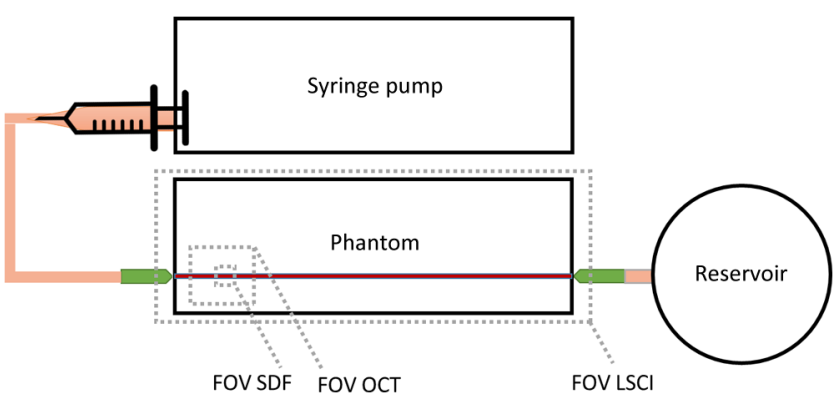

Fig. 1 Schematic figure of the setup with a syringe pump (Harvard Apparatus), a 10-mL syringe, tubing (pink), blunt entry/exit needles (green), a simulated vessel (red), and a reservoir. The FOV and localization of measurements are indicated by dotted lines for $(\mathrm{LSCl} 10 \mathrm{~cm} \times 7.5 \mathrm{~cm})$, OCT $(10 \mathrm{~mm} \times 10 \mathrm{~mm})$, and SDF $(0.94 \mathrm{~mm} \times$ $0.75 \mathrm{~mm})$.

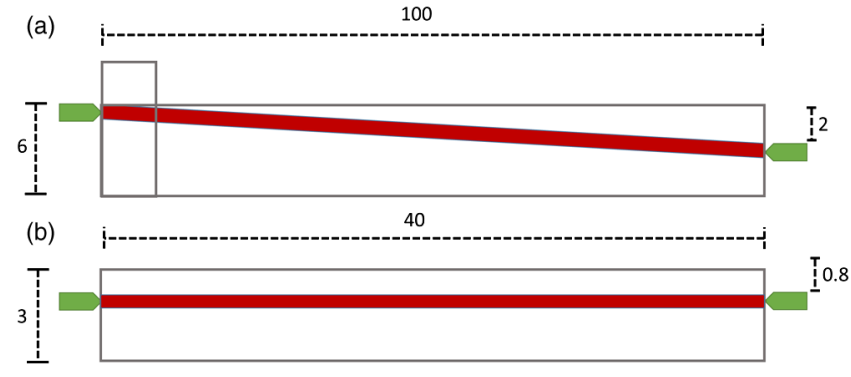

Fig. 2 Schematic figure of phantom A with tilted vessels (red) (50 and $100 \mu \mathrm{m}$ diameter) and phantom B containing a horizontal vessel (400 $\mu \mathrm{m}$ diameter) and connective blunt needles (green). Dimensions are given in $\mathrm{mm}$.

angle of $1.15 \mathrm{deg}$ with respect to the surface, creating a variety in imaging depth. In phantom B, one horizontal vessel with a diameter of $400 \mu \mathrm{m}$ was positioned with the center of its lumen at $800 \mu \mathrm{m}$ below the phantom surface (Fig. 2).

To manufacture the phantoms, leads of tungsten wire with diameters of 50 and 100 or $400 \mu$ m were placed in an aluminum container. The tungsten wire was passed through a blunt needle (NORDSON EFD 18 Gauge) that was embedded in the phantom wall. Next, silicone and elastomer $\left(\right.$ Sylgard $^{\circledR} 184$ Silicone Elastomer DOW/Corning) were added in this container at a ratio of $1: 10$. Following the recipe described by de Bruin et al., ${ }^{15}$ $0.2 \%$ titanium dioxide $\left(\mathrm{TiO}_{2}\right)$ was added to mimic human tissue optical scattering properties $\left(\mu_{\mathrm{t} 800 \mathrm{~nm}}=4 \mathrm{~mm}^{-1}\right)$. After consolidation of the phantom in an oven at $60^{\circ} \mathrm{C}$ for $8 \mathrm{~h}$, the thin tungsten and blunt needle were both carefully pulled out of the phantom, creating phantom vessels without the need for glass capillaries. Because the embedded needles were filled with silicone during the manufacturing process, new blunt needles were placed at both ends to allow connection of tubing to the phantom. The total length of the 50- and $100-\mu \mathrm{m}$ vessels in phantom A was $\sim 100 \mathrm{~mm}$, whereas the $400-\mu \mathrm{m}$ vessel in phantom $B$ was $\sim 40 \mathrm{~mm}$ in length. The distance between the 50 - and $100-\mu \mathrm{m}$ vessels in phantom A was $\sim 10 \mathrm{~mm}$.

\subsubsection{Pump}

A stable 20-mL PHD 200 infusion syringe pump (Harvard Apparatus, Holliston) was used to perfuse the vessels. After heparinizing the system (20 units $/ \mathrm{mL}$ ), whole blood from a healthy volunteer was collected in a $20-\mathrm{mL}$ syringe. Pump flow accuracy and variability were checked at $5 \mathrm{~mL} / \mathrm{h}$ with a mini CORI-FLOW ${ }^{\mathrm{TM}}$ mass flow controller (Bronkhorst hightech B.V., Ruurlo, The Netherlands) resulting in a measured flow of $4.97 \pm 0.03 \mathrm{~mL} / \mathrm{h}$. During the experiments, blood was pumped through the phantom and collected in a reservoir.

\subsection{Data Acquisition Modalities and Analysis}

LSCI, OCT, and SDF were employed to image blood vessels and measure blood flow velocity and vessel diameter in the phantoms. All imaging modalities were manually positioned perpendicularly to the phantom surface (Fig. 1). For each diameter $(50,100$, and $400 \mu \mathrm{m})$, we calculated the pump flow settings to produce average velocities in the vessels of the phantom of $0,1,5,10$, and $20 \mathrm{~mm} / \mathrm{s}$, matching the range of velocities observed in human microcirculation. ${ }^{16}$ 


\subsubsection{Laser speckle contrast imaging}

The LSCI device is a noncontact near-infrared imaging instrument with a large FOV (MoorFLPI-1 ${ }^{\mathrm{TM}}$, Moor Instruments, Devon, United Kingdom) operating at a wavelength of $785 \mathrm{~nm}$. The FOV is adjustable with a working distance between 15 and $45 \mathrm{~cm}$, typically operated to a maximum scan area of $15 \times 20 \mathrm{~cm}^{2}$. We used an FOV approximating $10 \times 7.5 \mathrm{~cm}^{2}$ with a total pixel area of $768 \times 576$.

The laser head to target distance was fixed at $24 \mathrm{~cm}$ above the phantom using an adjustable arm and tabletop clamping system. Optimal focus, zoom, and polarization were set prior to data acquisition according to manufacturer recommendations. Grayscale images were acquired with the shortest available exposure time of $4.2 \mathrm{~ms}$ at a frame rate of 25 frames/s.

The measured variation of speckle in the grayscale images and in time is translated into a single-color-coded "flux image" by the apparatus through a propriety algorithm. Here, the flux images were computed using 25 grayscale images, resulting in 1 flux image/s.

For each combination of the vessel diameter and flow velocity setting, 10 flux images were acquired (total acquisition time $10 \mathrm{~s}$ ) of which the 5 flux images with visible contrast between the vessel and surrounding area were used in subsequent analysis.

After selecting the region of interest (vessel-area) of $775 \times$ 13 pixels for phantom $\mathrm{A}$ and $282 \times 10$ pixels for phantom $\mathrm{B}$, assessment of perfusion was obtained from a windows-based MoorFLPI analysis software (MoorFLPI Review V4.0, Moor Instruments, Devon, United Kingdom) and expressed in mean \pm standard deviation laser speckle perfusion units (LSPU).

\subsubsection{Optical coherence tomography}

The OCT system is a noncontact, medium FOV, near-infrared depth-resolved imaging modality (Santec OCT system IVS2000, Santec USA Corporation, Hackensack) operating at a wavelength of $1310 \mathrm{~nm}$ at $50 \mathrm{kHz}$ linerate. The FOV of the handheld probe head is adjustable, typically operated to a maximum area of $2 \times 2 \mathrm{~cm}^{2}$. The axial resolution is $12 \mu \mathrm{m}$ and the lateral resolution is $50 \mu \mathrm{m}$ (beam waist $25 \mu \mathrm{m}$ ). The OCT system was placed and fixed $6 \mathrm{~cm}$ above the phantom using an adjustable arm and tabletop clamping system. Phantom position was set just under zero-delay before data acquisition. In-house custombuilt software designed using LabVIEW (LabVIEW 2013, National Instruments) was used to measure the vessel diameter $(\mu \mathrm{m})$ and decorrelation-time $\tau(\mathrm{ms})$ in the vessel.

Three-dimensional (3-D) scans were acquired at a scan area $(x, y, z)=(3.0,3.0, z=2.5) \mathrm{mm}$ and pixel range of $(x, y, z)=(400,400,400)$ pixels. Vessel diameters were measured using an on-screen measurement tool (Fig. 4) in five cross-sectional OCT images per 3-D OCT dataset. Diameters measured in $75 \mathrm{~B}$-scans are expressed as mean $\pm \mathrm{SD}$.

M-mode scans, A-scans at the same location in time $(n=15)$, were made at the center of the vessel at each pump setting ( 0 to $20 \mathrm{~mm} / \mathrm{s}$ ) to measure OCT speckle decorrelation-times that are inversely proportional to velocity. ${ }^{17}$

Decorrelation time was determined from 400 M-mode images of $400 \mathrm{~A}$-scans each measured in the region identified as flow channel. First, the amplitude versus time trace at each depth was extracted (total duration $8 \mathrm{~ms}$ per M-scan). Next, from each time-trace, the autocovariance function was calculated. A Gaussian function $\mathrm{g}(t)=\exp \left[-(t / \tau)^{2}\right]$ was fitted to the average of the ACF over $400 \mathrm{M}$-scans. Here, $t$ is time lag and $\tau$ is the decorrelation time. Since at high velocity the ACF falls to zero rapidly, in practice only 150 to 250 time points (out of the 400 available) were used. Finally, mean \pm SD of the inverse decorrelation times corresponding to the vessel depth position were extracted. Weiss et al. ${ }^{17}$ demonstrated that for single scattering, the relation between OCT determined decorrelation time $(\tau)$ and flow velocity $(V)$ is $\tau^{-1}=\omega^{-1} V$ where $\omega$ is the beam waist ( $25 \mu \mathrm{m}$ in our system, defined and measured as the lateral distance at which the field amplitude of the Gaussian beam drops to $e^{-1} \sim 0.37 \times$ its maximum on-axis value).

\subsubsection{Sidestream dark-field imaging}

The SDF device is a handheld, direct contact, small FOV visible light modality (Microvision Medical, Amsterdam, The Netherlands) operating at a wavelength of $550 \mathrm{~nm}$. The FOV is $0.94 \times 0.75 \mathrm{~mm}$ imaged by $720 \times 576$ pixels, with a maximum focus depth of $400 \mu \mathrm{m}$.

Optimal focus was set at $50 \mu \mathrm{m}$ before data were obtained. Images were acquired at a frame rate of 25 frames/s for a total of $10 \mathrm{~s}$, this was repeated three times per vessel diameter/pump setting combination.

For analysis, the magnification of the camera was determined using a special grid-calibration cylinder (Microvision Medical, Amsterdam, The Netherlands) resulting in a horizontal pitch of $1.48 \mu \mathrm{m} /$ pixel and a vertical pitch of $1.35 \mu \mathrm{m} /$ pixel. After selecting the center of the vessel, the vessel diameter $(\mu \mathrm{m})$ was determined using an Automated Vascular Analysis software package (AVA version 3.2, Microscan B.V., Amsterdam, The Netherlands). Using the same software, blood flow velocity $(\mu \mathrm{m} / \mathrm{s})$ was measured with a space-time diagram. ${ }^{18}$ Vessel diameters and blood flow velocity are presented as mean \pm SD.

\section{Results}

\subsection{Laser Speckle Contrast Imaging}

LSCI was not able to image vessels of 50 and $100 \mu \mathrm{m}$ in diameter, up to a minimum depth of $100 \mu \mathrm{m}$ below the surface of phantom A, for all blood flow settings [Figs. 3(a) and 3(b)].

In contrast, the $400-\mu \mathrm{m}$ vessel that was positioned at $800 \mu \mathrm{m}$ below the surface of phantom B was visible with LSCI [Fig. 3(c)]. Also, as shown in Fig. 3(d), LSPU increased with induced blood flow in the vessel. The LSPU seemed to saturate for velocities exceeding $5 \mathrm{~mm} / \mathrm{s}$.

\subsection{Optical Coherence Tomography}

OCT could clearly visualize the $50-\mu \mathrm{m}$ vessel [Fig. 4(a)], the $100-\mu \mathrm{m}$ vessel [Fig. 4(b)], and the $400-\mu \mathrm{m}$ vessel in crosssectional images [Fig. 4(c)].

The phantom was imaged slightly under an angle to avoid specular reflectance. The vessels were visible as circular regions in the middle of the cross-sectional OCT images. Figure 4(d) demonstrates the linear relation between $1 / \tau$ and the induced blood velocity for the $100 \mu \mathrm{m}$ in red $\left(R^{2}=0.95\right)$ and $400 \mu \mathrm{m}$ in blue $\left(R^{2}=0.99\right)$ vessel, although the slope was different both cases. Comparing the expected and, based on single scattering theory, measured velocities, an overestimation of average blood flow velocities is clearly observed.

Since the $50-\mu \mathrm{m}$ vessel is depicted by five pixels, this vessel was difficult to localize [Fig. 4(a)]. Therefore, the M-scans 
(a)

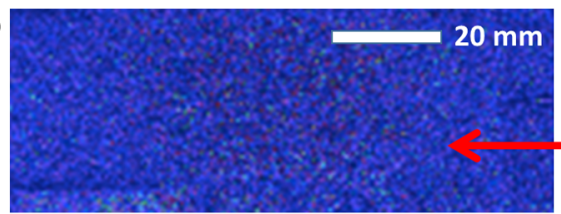

(b)

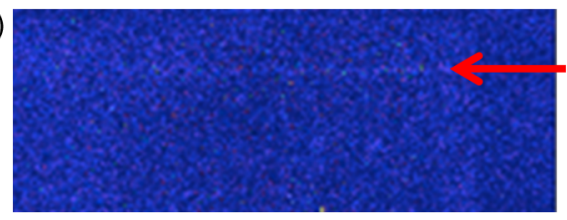

(c)

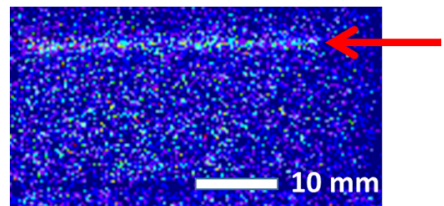

(d)

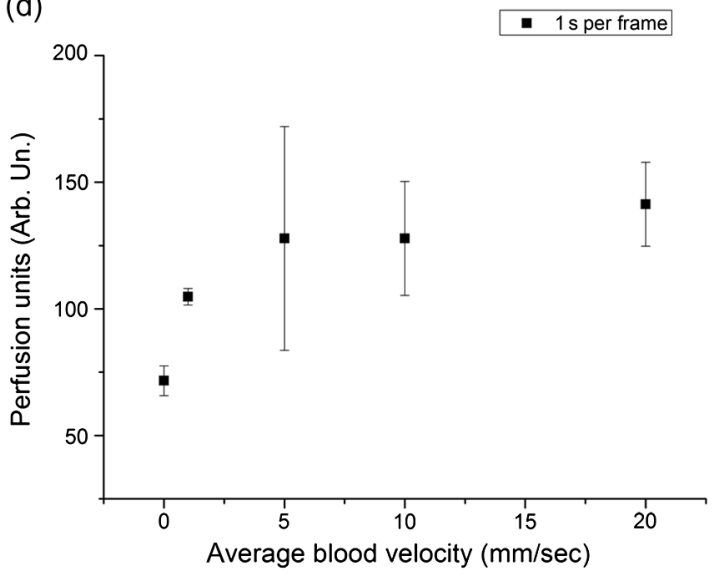

Fig. $3 \mathrm{LSCl}$ images of (a) 50, (b) 100, and (c) $400 \mu \mathrm{m}$ vessel diameter in a phantom with flowing blood at a velocity of $20 \mathrm{~mm} / \mathrm{s}$. Only the $400-\mu \mathrm{m}$ vessel was visible on the blue LSCl image (vessels indicated by red arrows). (d) The set average blood flow velocity compared to measured perfusion units in the $400-\mu \mathrm{m}$ vessel for $1 \mathrm{~s}$ per frame. Perfusion units increased nonlinearly with higher blood flow velocities.

(a)

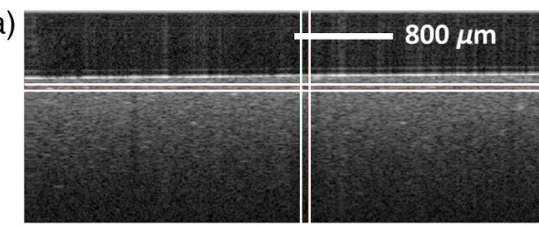

(b)

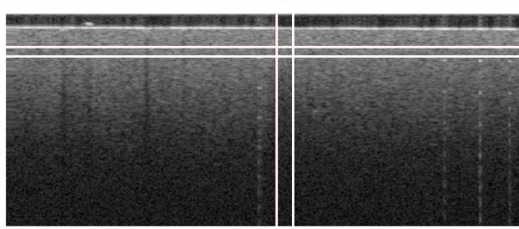

(c)

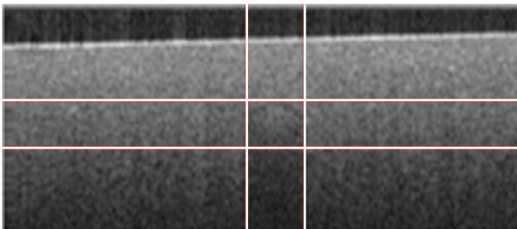

(d)

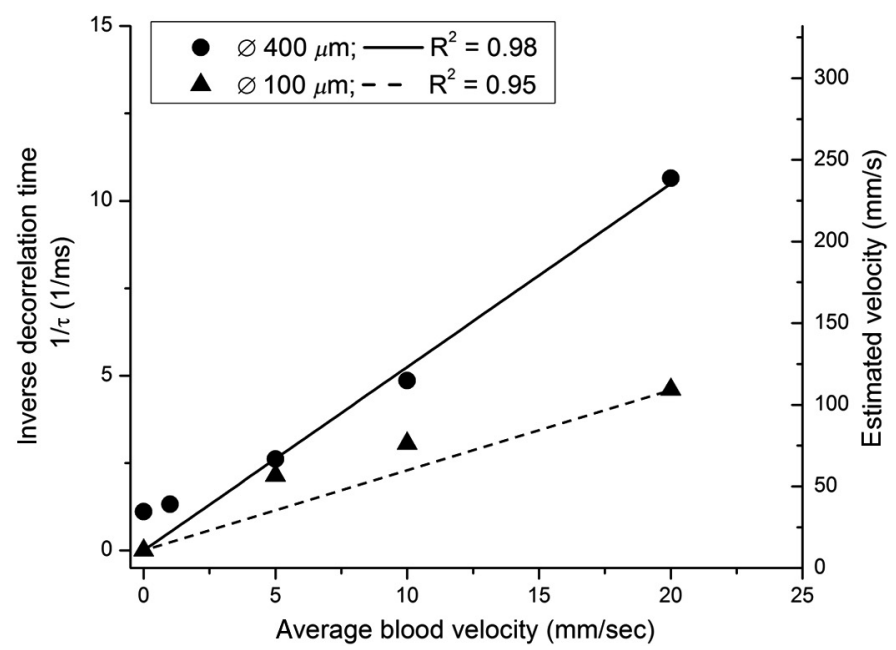

Fig. 4 OCT images of (a) 50, (b) 100, and (c) $400 \mu \mathrm{m}$ cross-sectional view showing lumen inside the phantoms, with red crosshairs delineating the vessel boundaries. (d) The measured decorrelation time versus the set average blood flow velocity for the $100-\mu \mathrm{m}$ vessel in red $\left(R^{2}=0.95\right)$ and the $400-\mu \mathrm{m}$ vessel in blue $\left(R^{2}=0.99\right)$ : $1 /$ decorrelation time increased linearly with the blood velocity. The righthand side axis shows the estimated velocity based on the single scattering model by Weiss et al. ${ }^{17}$

location of this vessel was not accurately positioned, so the decorrelation time was not measurable.

\subsection{Sidestream Dark-Field Microscopy}

SDF was able to visualize the 50- $\mu \mathrm{m}$ vessel [Fig. 5(a)] and the $100-\mu \mathrm{m}$ vessel [Fig. 5(b)] in phantom A down to a maximum depth of $200 \mu \mathrm{m}$, albeit with a lower contrast. Vessel diameter could be measured with the AVA3.2 software.

Also, the blood in the $400-\mu \mathrm{m}$ vessel that was positioned at a depth of $800 \mu \mathrm{m}$ was visible compared to the surroundings [Fig. 5(c)]. For all diameters, blood flow velocities could not be measured using whole blood as a flowing medium.
To check if SDF was feasible to measure velocity after hemodilution, we diluted blood up to $10 \%$ and generated images in the $100-\mu \mathrm{m}$ vessel. Here, it was possible to measure average blood flow velocities. However, due to the frame rate of the CCD camera (25 frames/s) and the FOV of $\sim 1 \mathrm{~mm}^{2}$, only velocities up to $1 \mathrm{~mm} / \mathrm{s}$ could be measured. Figure 5(d) presents the relation between measured and actual velocities.

\subsection{Comparison of Vessel Diameter Measurements}

Vessel diameters of the three phantom vessels were measured precisely with OCT and SDF (small SD, Fig. 6), although for the $400 \mu \mathrm{m}$, the average diameter was smaller than the expected dimensions. 
(a)

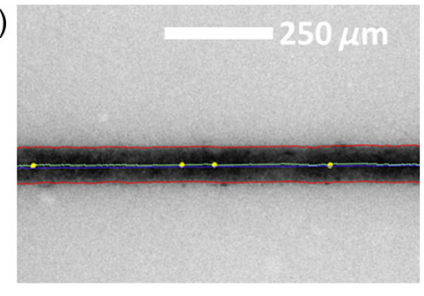

(b)

(c)

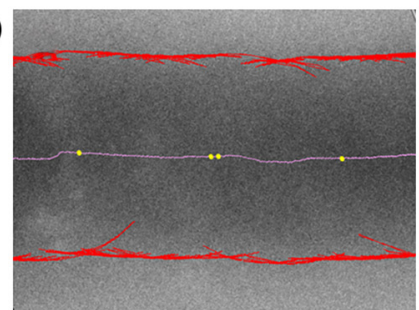

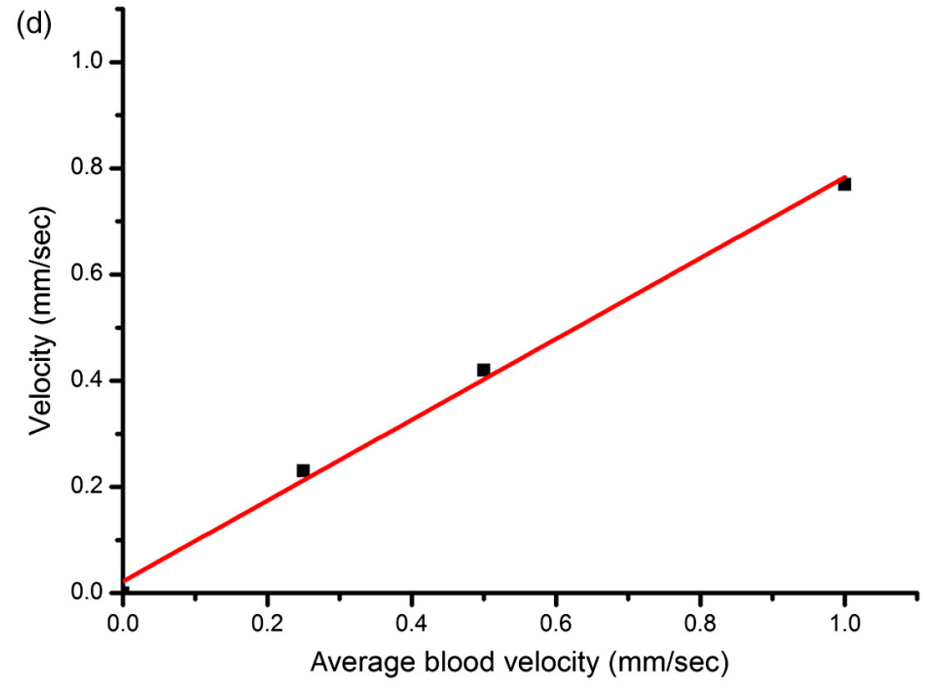

Average blood velocity $(\mathrm{mm} / \mathrm{sec})$

Fig. 5 SDF frames of (a) 50, (b) 100, and (c) $400 \mu \mathrm{m}$ phantoms with vessels filled with full blood en face view. (d) The relation between measured and set average velocity for diluted blood (1:10) in the $100-\mu \mathrm{m}$ vessel. Because of the high reproducibility error bars are not visible. Fit: $\left(R^{2}=0.98\right.$, intercept $=0.04$, and slope $=0.75$ ).

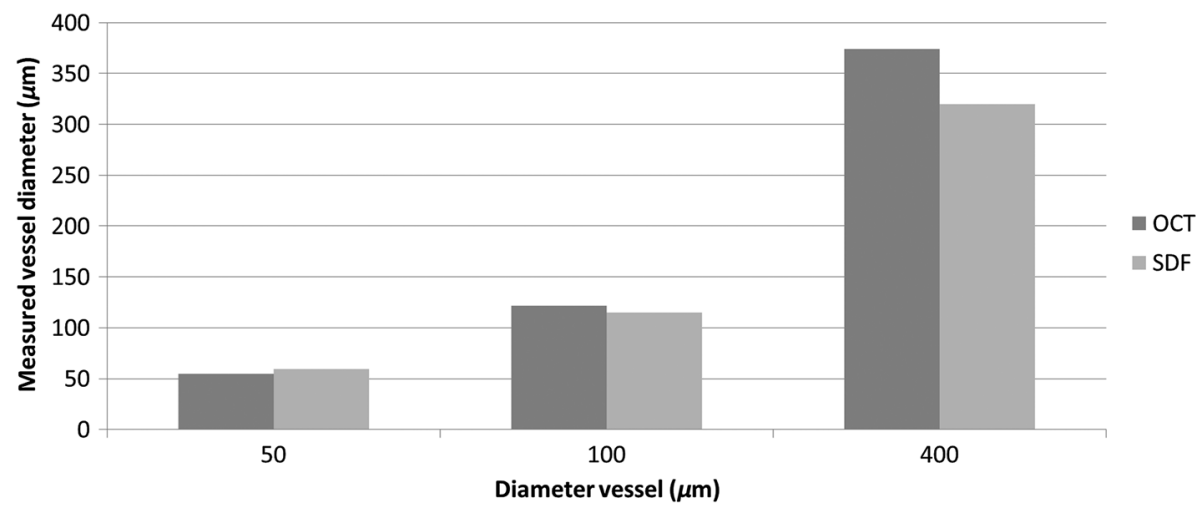

Fig. 6 Measured vessel diameters (average \pm SD) with OCT (blue) and SDF (orange).

\section{Discussion}

This study demonstrates the feasibility of commercially available LSCI, OCT, and SDF systems to visualize perfusion and the validity to measure blood perfusion-related parameters as vessel diameter and blood flow velocity in an in vitro phantom model. Thereto, whole blood was perfused through microvascular constructs free of glass capillary layering in an attempt to closely mimic human soft tissue. Vessel diameters could be precisely determined by OCT and SDF. Blood flow velocities could be assessed as well. LSCI measured flow in perfusion units for the $400-\mu \mathrm{m}$-diameter vessel only, with a nonlinear dependence on velocity. OCT amplitude inverse decorrelation time $(1 / \tau)$ scaled linearly with the blood velocity for the 100- and $400-\mu$ m-diameter vessel. SDF was not able to measure blood flow velocity using whole blood; only with diluted $10 \%$ blood SDF could quantify low blood flow velocity (0 to $1 \mathrm{~mm} / \mathrm{s}$ ) in the $100-\mu \mathrm{m}$-diameter vessel.
LSCI is a technology widely studied in patients, yet validation and interpretation of LSPU and imaging of single vessel diameters with the MoorFLPI system are challenging. Speckle dynamics are influenced not only by motion but also by laser stability, bulk motion, integration time, and the concentration of scatterers within the probed volume. These factors inhibit absolute quantification of perfusion so far. As a result, arbitrary LSPU are used. The link of LSPU to quantitative parameters such as blood flow, velocity, and perfusion yet remains unclear. ${ }^{19}$ Nadort et al. combined LSCI with SDF with encouraging results that yield a quantitative measurement of blood flow velocity, although it is not yet clinically applicable because of instrumentational challenges. ${ }^{20}$ Using multiple exposure times, LSCI was capable of retrieving speckle decorrelation times that can be related to blood flow velocity through a combination of LSCI and SDF microscopy on in vitro and in vivo flow samples. ${ }^{21}$ The conventional, linear scaling between blood 
flow velocity $(V)$ and inverse decorrelation time $(1 / \tau)$ was expressed as $1 / \tau=A(N) \alpha V$; where $\alpha$ is a proportionality constant that depends on the optical properties of the scatterers and likely on image geometry; $A(N)$ is a factor accounting for faster decorrelation due to higher $N$ 'th-order scattering occurring in the flow region. The factor $A(N)$ depends on the expected number of scattering events in a vessel; for a vessel of diameter $d$ and particles with scattering coefficient, as a rule of thumb, for LSCI illumination and detection it was found that $N=1.2 \mu_{\mathrm{s}} d .{ }^{20}$ The link of LSPU to quantitative parameters such as blood flow, velocity, and perfusion yet remains unclear. ${ }^{19}$ The LSPU values reported here are computed using a propriety algorithm by the manufacturer.

Our study demonstrates a lack in LSCI visibility for vessels of the 50 and $100 \mu \mathrm{m}$ diameters using our Moor FLPI system at the current FOV. Also, LSPUs appeared to saturate for velocities exceeding $5 \mathrm{~mm} / \mathrm{s}$ with a stark increase in standard deviation. The conversion of spatial speckle contrast to LSPU is propriety information of the manufacturer. Nevertheless, low speckle contrast relates to high velocities and, we assume, to high LSPU values as well. Therefore, small fluctuations in low spatial contrast values (raw measurement) may lead to large fluctuations in corresponding LSPU. We applied the approach by Nadort et al. ${ }^{20}$ to first determine estimates of the speckle decorrelation time associated with our experimental geometry and use Eq. (3) of Ref. 19 to convert to estimates of contrast. For velocities of $0.01,1,5,10$, and $20 \mathrm{~mm} / \mathrm{s}$, this leads to contrast values of $0.93,0.21,0.09,0.07$, and 0.05 . The last three values are all below 0.1 with less than a factor of 2 difference in contrast for a factor 4 difference in velocity. This problem can be reduced using shorter exposure times at the higher velocities. Unfortunately, this is not possible with our current instrument.

On the other hand, the wide-field color-coded LSCI image is an easy way for clinicians to assess the perfusion differences in tissue intraoperatively and is therefore an advantage of this technique in providing an on the fly rapid perfusion profile projection.

OCT-based imaging of vascular dynamics has been studied in different clinical settings. A distinction can be made between analysis of amplitude and phase data versus amplitude data only. By using phase and amplitude data, Doppler OCT quantifies the motion-induced phase difference of light scattered from flowing RBCs with respect to static tissue. When the angle between the incidence (and observation) angle and the velocity direction is known or measured, the flow velocity $(\mathrm{mm} / \mathrm{s})$ can be determined because Doppler OCT is only sensitive to the projection of the velocity vector parallel to the beam direction. Optical microangiography segments the measured data based on frequency shift, i.e., voxels where nonzero frequency shifts measured are assigned to the microcirculation. ${ }^{22}$ Methods based on intensity signal only are also explored, with the specific advantage of compatibility with commercially available OCT systems, which usually do not provide phase data. A straightforward approach to determine the presence of hemodynamics calculates the speckle variance between subsequent B-scans ${ }^{23}$ or determines the decorrelation time from M-mode images as in this study. ${ }^{17}$ In this study, we found a linear relation between inverse decorrelation time and the average blood velocity in the channel $(R>0.95)$ albeit with a much higher proportionality constant than predicted, i.e., the imaging beam waist. Multiple scattering in the blood vessel leads to faster decorrelation, which makes scaling of inverse decorrelation time to velocity a challenging

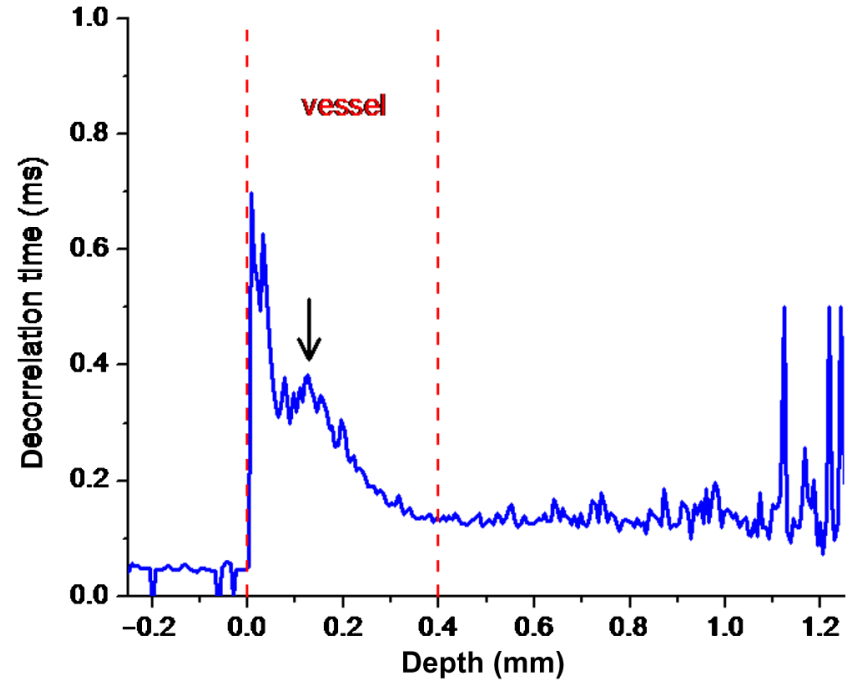

Fig. 7 Decorrelation time versus depth for the phantom with a $400-\mu$ m-diameter channel, at pump flow setting of $5 \mathrm{~mm} / \mathrm{s}$. Dashed vertical bars depict the channel boundaries. The horizontal depth axis is shifted so that the top channel boundary as found by visual inspection of the OCT image is at zero position. The arrow marks the location where the decorrelation time $(1 / \tau C=2.6 \mathrm{~m} / \mathrm{s})$ was estimated.

task. For single scattering, the relation between OCT determined decorrelation time $(\tau)$ and flow velocity $(V)$ is $\tau^{-1}=\omega^{-1} V$, where $\omega$ is the beam waist. ${ }^{17}$

Using the result of Nadort et al., ${ }^{20}$ we assume that additional decorrelation caused by multiple scattering can be modeled by incorporating scaling factor $A(N)$ in $\tau^{-1}=A(N) \omega^{-1} V$ where $N$ is the number of scattering events. We estimate the number of scattering events experienced by photons returning from the vessel center as $N \sim \mu_{\mathrm{s}} d$ (accounting for travel to and from the vessel center), with $\mu_{\mathrm{s}}$ is the scattering coefficient and $d$ is the diameter. From Boschaart et al., ${ }^{24}$ the scattering coefficient $\mu_{\mathrm{s}}$ at $1.3 \mu \mathrm{m}$ is $\sim 45 \mathrm{~mm}^{-1}$. Thus, the expected number of scattering events experienced by detected photons from the vessel center is $N=18$ and for the 400- and 100- $\mu$ m diameter vessels. Values of $A(N)$ derived from our measured data (by the regression slope of the estimated velocity versus actual velocity) are $A(N) \sim 13$ and $A(N) \sim 6$. Based on our work in Ref. 20, $A(N) \sim 7$ and $A(N) \sim 3$ are predicted. This leads us to believe that the difference in inverse decorrelation times between vessels of different diameters can be attributed to multiple scattering by whole blood in these blood vessels.

Figure 7 shows the decorrelation time versus depth measured from the experiment using a $400-\mu \mathrm{m}$ channel and $5-\mathrm{mm} / \mathrm{s}$ flow velocity.

The decrease of decorrelation time with depth in the channel is again attributed to multiple forward scattering. Moreover, signals from well below the phantom vessel show measurable decorrelation times where a stationary signal is expected. This is readily explained by photons interacting with moving particles in the vessel on their way to and from the static scattering site. Consequently, translating decorrelation times directly into velocities is difficult, because the number of scattering events has to be estimated.

Moreover, we did not take the effect of the velocity profile into account. Because of the non-Newtonian flow behavior of whole blood, it is likely that the flow profile is nonparabolic. 
Moger et al. indeed described a blunt flow profile for blood in phantom vessels with time-domain Doppler OCT. ${ }^{25,26}$ Quantitative flow analysis using the decorrelation time is therefore still not clinically employable. Vessel diameters, however, are measured precisely with OCT, given the expected depth resolution. For the largest, deeper lying vessel determination of specifically the lower boundary was challenging. Since a mischaracterization by a single pixel leads to a $12-\mu \mathrm{m}$ difference in estimated diameter, we believe this is the main cause for our underestimation of the diameter of the $400-\mu \mathrm{m}$ vessel.

$\mathrm{SDF}$ is a handheld-system that requires contact with tissue through using a disposable sterile cap. The amount of light and focus position has to be regulated by hand, which is difficult considering the handheld FOV of $0.94 \times 0.75 \mathrm{~mm}^{2}$. SDF is the only optical technology that attempts to measure red blood cell velocity in $\mathrm{mm} / \mathrm{s}$.

To our knowledge, this is the first time that a flow phantom without a glass capillary is used to validate blood flow velocity with SDF. This reduces associated artifacts, such as high intensity reflection from the phantom-glass interface and lensing by the curved surfaces.

The blood flow velocity analysis is based on tracing RBCs, or clusters of RBCs, in consecutive frames at the vessel center line. The tracing results in a space-time diagram in which the steepness of the line pattern accounts for the velocity. With increasing vessel thickness $(>\sim 50 \mu \mathrm{m})$ or hematocrit, the visibility of alternating cells and plasma gaps becomes decreasingly visible. High velocities also result in a blurred space-time diagram. Tracing was however not possible in the whole blood experiments. In order to mimic these clusters of RBCs, the whole blood had to be diluted.

The velocity sensitivity depends on the frame rate of the CCD camera, which is fixed at 25 frames/s. Because of this frame rate, it was only possible to measure velocities below $1 \mathrm{~mm} / \mathrm{s}$, which is less than reported by others $(2.0 \mathrm{~mm} / \mathrm{s}){ }^{21}$ Measurements showed a linear correlation with expected blood flow velocities $(R=0.99)$ albeit at lower values than the expected maximum velocity. We attribute this to inaccurate manual selection of the vessel center line along which the spacetime diagram is calculated.

Due to the need for RBC clusters and the limited range of velocity measurement, SDF is only suitable for quantifying the flow in capillaries and small arterioles. A small underestimation of the determined velocity was found for SDF, which is most likely due to an incorrect assumption of the flow profile, that can range from parabolic to uniform, or a small deviation during the calibration of the magnification of the system. The vessel diameters were measured accurately and precisely, to a depth of $200 \mu \mathrm{m}$. The quality of the images is imaging-depth depended, and therefore, the $400-\mu \mathrm{m}$ vessel, located at a depth of $800 \mu \mathrm{m}$ beyond the achievable focal plane, could not be observed.

All three optical instruments used in this study do not require an extrinsic contrast mechanism and are commercially available and on the verge of clinical translation. Therefore, physicians could implement these technologies in their clinical practice. Other optical techniques that have this potential are laser Doppler flowmetry (LDF), laser Doppler imaging (LDI), fluorescence imaging, and thermographic or near-infrared imaging. Considering the heterogeneity of microcirculation, a disadvantage of LDF is the use of point measurements and the lack of imaging. ${ }^{27}$ Scanning LDF was developed to overcome this problem; however, imaging time is very slow. Although parallel and faster imaging has been demonstrated, ${ }^{28}$ LDI has been replaced by laser speckle imaging, which images in a much higher speed. Fluorescence imaging requires an extraintervention with a fluorophore (e.g., indocyanine green) to image vessels. $^{29}$ Temperature changes occur at a later stage than changes in flow, which makes thermographic imaging a less ideal technology as well. ${ }^{30}$

Finally, tissue phantoms as used in this study are previously described in the literature. ${ }^{15}$ In this study, we meet 8 of the 12 criteria for the "ideal phantom" stated by Pogue and Patterson: ${ }^{31}$ (1) wavelength dependence of properties that are similar to tissue, (2) material properties that are stable over time, (3) index of refraction close to tissue, (4) incorporate regions with different optical properties using single vessels with whole blood, (5) incorporate flow in the phantom, (6) ease of manufacturing, (7) inexpensive to produce, and (8) easily transported among different sites. The optical properties that were used in this phantom study are optimized for near-infrared light techniques. Human capillaries have a diameter ranging on average between 5 and $10 \mu \mathrm{m}$; in this report, $50 \mu \mathrm{m}$ was the smallest vessel that was integrated in the phantom. In the future, clearly a capillary phantom network is needed for biologically accurate validating perfusion parameters.

\subsection{Limitations and Clinical Implications}

We anticipated that these commercially available optical techniques could be valuable in the clinical setting for perfusion imaging during surgery. However, none of them measure perfusion in the desired parameter of flow per unit of tissue mass $\left(\mathrm{mL} \cdot \mathrm{min}^{-1} \cdot \mathrm{g}^{-1}\right.$ tissue). Because it is the only volumetric depth-resolved imaging technology, OCT has the potential to measure this parameter.

LSCI and OCT were not able to measure blood flow velocity in the smallest vessel $(50 \mu \mathrm{m})$, whereas SDF was able to detect a $50-\mu \mathrm{m}$ vessel. SDF, however, could only measure small blood flow velocities up to $1 \mathrm{~mm} / \mathrm{s}$ in diluted blood (10\%). Also, SDF was not able to measure the diameter of the $400-\mu \mathrm{m}$ vessel because it lacked in imaging depth. Therefore, the choice of modality for perfusion in imaging in de clinic in this stage will depend on the clinical question: what is the desired probed volume, lies the interest in superficial or deeper vessels, which resolution is required? For local imaging of subsurface capillaries, SDF is favorable; alternatively, for local in deeper imaging of capillaries, arteries, and veins, the OCT could be the first choice; finally, for imaging of a large surface area such as a whole organ surface total perfusion, LSCI could be used.

A technique in which macroscopic and microscopic imaging is combined would be ideal. Macroscopic imaging will give the clinicians a value for the overall tissue perfusion. Microscopic resolution will yield information regarding blood flow in tissue capillaries, which are important for tissue vitality because of the exchange of oxygen and nutrients. An ideal imaging technique further acquires images and measures perfusion (related parameters) in real-time during surgery, enabling the surgeon to immediately adapt his surgical approach toward optimal perfusion.

\section{Conclusion}

We present quantitative perfusion imaging with LSCI, OCT, and SDF Microscopy in a tissue-like flow phantom with microvessels. LSCI, OCT, and SDF were all able to visualize flowing 
blood. The measured perfusion-related parameters by LSCI scale nonlinearly with the blood velocity. LSCI is more useful to assess differences in perfusion in a large FOV. OCT was able to quantify deep-lying vessel diameters precisely and could assess blood flow velocity in terms of inverse OCT speckle decorrelation time at a higher range of velocities than SDF. Scaling for multiple scattering effects by moving red blood cells remains challenging. Velocity estimation of whole blood using SDF was not feasible; however, for diluted blood flow, velocity was linear with the input velocity up to a velocity of $1 \mathrm{~mm} / \mathrm{s}$. The diameters of narrow superficial vessels were accurately measured.

\section{Disclosures}

Dr. Jansen, Dr. de Bruin, Dr. Faber, Dr. Dobbe, Dr. Heeg, Dr. Milstein, and Dr. Strackee have nothing to disclose.

\section{Acknowledgments}

The authors would like to thank C. Kess for his contribution to this study. Moreover, they would like to thank ZonMw for their financial support and Insitute Quantivision for their support in trial conception. Dr. van Leeuwen reports grants from ZONMW, nonfinancial support from Quest innovations, from null, during the conduct of the study; other from PA imaging $\mathrm{BV}$, grants and nonfinancial support from Lionix, grants and nonfinancial support from Xiophotonics, grants and nonfinancial support from Ninepoint, outside the submitted work; In addition, Dr. van Leeuwen has a patent "Combined Raman Spectroscopy Optical Coherence Tomography (RS-OCT) System and Applications of the Same" issued, a Patent "Common detector for combined Raman spectroscopy-optical coherence tomography" issued, a Patent "Arthroscopic instrument assembly, and method of localizing musculoskeletal structure during Athroscopic surgery" issued, a patent "Flow cytometry method for determination of size and refractive index of substantially spherical single particles and calibration method suitable for use with such a flow cytometry method" pending, a patent "High Wavenumber Raman Spectroscopy and Applications of Same pending, and a patent Common-Path Integrated Low Coherence Interferometry System and Method Therefore" pending.

\section{References}

1. C. L. Kerrigan, "Skin flap failure: pathophysiology," Plast. Reconstr. Surg. 72(6), 766-774 (1983).

2. C. Salgado et al., "Postoperative care and monitoring of the reconstructed head and neck patient," Semin. Plast. Surg. 24(3), 281-287 (2010).

3. A. K. Wong et al., "Analysis of risk factors associated with microvascular free flap failure using a multi-instituational database," Microsurgery 35(1), 6-12 (2010).

4. P. van Hagen et al., "Preoperative chemoradiotherapy for esophageal or junctional cancer," N. Engl. J. Med. 366(22), 2074-2084 (2012).

5. H. Iida et al., "Measurement of absolute myocardial blood flow with $\mathrm{H}_{215} \mathrm{O}$ and dynamic positron-emission tomography. Strategy for quantification in relation to the partial-volume effect," Circulation $\mathbf{7 8}(1)$, 104-115 (1988).

6. C. Cuenod et al., "Early changes in liver perfusion caused by occult metastases in rats: detection with quantitative CT," Radiology 218(2), 556-561 (2001).

7. L. Y. Hsu et al., "Quantitative myocardial perfusion analysis with a dualbolus contrast-enhanced first-pass MRI technique in humans," J. Magn. Reson. Imaging 23(3), 315-322 (2006).
8. B. White et al., "In vivo dynamic human retinal blood flow imaging using ultra-high-speed spectral domain optical coherence tomography," Opt. Express 11(25), 3490-3497 (2003).

9. C. Jung et al., "Evaluation of the sublingual microcirculation in cardiogenic shock," Clin. Hemorheol. Microcirc. 42(2), 141-148 (2009).

10. A. K. Dunn et al., "Dynamic imaging of cerebral blood flow using laser speckle," J. Cereb. Blood Flow Metab. 21(3), 195-201 (2001).

11. D. A. Boas and A. K. Dunn, "Laser speckle contrast imaging in biomedical optics," J. Biomed. Opt. 15(1), 011109 (2010).

12. P. T. Goedhart et al., "Sidestream dark field (SDF) imaging: a novel stroboscopic LED ring-based imaging modality for clinical assessment of the microcirculation," Opt. Express 15(23), 15101-15114 (2007).

13. D. Huang et al., "Optical coherence tomography," Science 254(5035), 1178-1181 (1991).

14. P. McCulloch et al., "No surgical innovation without evaluation: the IDEAL recommendations," Lancet 374(9695), 1105-1112 (2009).

15. D. M. de Bruin et al., "Optical phantoms of varying geometry based on thin building blocks with controlled optical properties," J. Biomed. Opt. 15(2), 025001 (2010).

16. J. Lindert et al., "OPS imaging of human microcirculation: a short technical report," J. Vasc. Res. 39(4), 368-372 (2002).

17. N. Weiss, T. G. van Leeuwen, and J. Kalkman, "Localized measurement of longitudinal and transverse flow velocities in colloidal suspensions using optical coherence tomography," Phys. Rev. E 88(4), 042312 (2013).

18. J. G. G. Dobbe et al., "Measurement of functional microcirculatory geometry and velocity distributions using automated image analysis," Med. Biol. Eng. Comput. 46, 659-670 (2008).

19. D. Briers et al., "Laser speckle contrast imaging: theoretical and practical limitations," J. Biomed. Opt. 18(6), 066018 (2013).

20. A. Nadort et al., "Quantitative blood flow velocity imaging using laser speckle flowmetry," Sci. Rep. 6, 25258 (2016).

21. A. Nadort et al., "Quantitative laser speckle flowmetry of the in vivo microcirculation using sidestream dark field microscopy," Biomed. Opt. Express 4(11), 2347-2361 (2013).

22. R. K. Wang and H. M. Subhash, "Optical microangiography: theory and application," in Microcirculation Imaging, pp. 246-254 (2012).

23. Y. M. Liew et al., "In vivo assessment of human burn scars through automated quantification of vascularity using optical coherence tomography," J. Biomed. Opt. 18(6), 061213 (2013).

24. N. Bosschaart et al., "A literature review and novel theoretical approach on the optical properties of whole blood," Lasers Med. Sci. 29(2), 453-479 (2014).

25. J. Moger et al., "Measuring red blood cell flow dynamics in a glass capillary using Doppler optical coherence tomography and Doppler amplitude optical coherence tomography," J. Biomed. Opt. 9(5), 982-994 (2004).

26. J. Moger et al., "The effect of multiple scattering on velocity profiles measured using Doppler OCT," J. Phys. D 38(15), 2597-2605 (2005).

27. M. Roustit et al., "Excellent reproducibility of laser speckle contrast imaging to assess skin microvascular reactivity," Microvasc. Res. 80(3), 505-511 (2010).

28. M. Draijer et al., "Twente optical perfusion camera: system overview and performance for video rate laser Doppler perfusion imaging," Opt. Express 17(5), 3211-3225 (2009).

29. J. V. Frangioni, "New technologies for human cancer imaging," J. Clin. Oncol. 26, 4012-4021 (2008).

30. W. J. Issing and C. Naumann, "Evaluation of pedicled skin flap viability by $\mathrm{pH}$, temperature and fluorescein: an experimental study," J. CranioMaxillofac. Surg. 24(5), 305-309 (1996).

31. B. W. Pogue and M. S. Patterson, "Review of tissue simulating phantoms for optical spectroscopy, imaging and dosimetry," J. Biomed. Opt. 11(4), 041102 (2006).

Sanne M. Jansen is a PhD student at the Academic Medical Center and works at the Department of Biomedical Engineering and Physics and the Department of Plastic, Reconstructive and Hand Surgery on optical imaging techniques in clinical diagnostics. Moreover, she is working as a medical doctor in the surgery department of the OLVG in Amsterdam.

Daniel M. de Bruin is appointed as staff member at the Department of Urology and as research associate at the Department of Biomedical 
Engineering and Physics of the AMC. In this combined position, he initiates new research lines which will ultimately result in a minimal invasive optical diagnosis and in novel focal treatment of (epithelial) cancers.

Dirk J. Faber is associate professor at the Academic Medical Center, University of Amsterdam. He works at the biophotonics group of the Department of Biomedical Engineering and Physics. The scope of his research is broadly be summarized in four topics: physical understanding of the optical signals, novel instrumentation, novel contrast mechanisms, and clinical application.

Iwan J. G. G. Dobbe is principal investigator at the Academic Medical Center, University of Amsterdam. His research scope is mechanical engineering, biomedical engineering, and electronic engineering. $\mathrm{He}$ is working on medical imaging and 3-D planning in surgery and has extensively studied velocity analysis.
Erik Heeg is a PhD student at the Dutch Institute for Clinical Auditing and is focusing on research in breast cancer and reconstruction.

Dan M. J. Milstein is a principal investigator at the Academic Medical Center, University of Amsterdam. He works in translational medicine.

Simon D. Strackee is a plastic and reconstructive hand surgeon at the Academic Medical Center. His work is focused on free flap reconstruction and hand surgery.

Ton G. van Leeuwen is a professor in biomedical physics and chairman of the new Department of Biomedical Engineering and Physics of the AMC in Amsterdam. At the BME and physics department we fuse engineering and physics with life sciences and clinical medicine, thereby providing further understanding of pathological processes and contributing to development and improvement of diagnostic and therapeutic tools. 